\title{
Multiphonon excitations and pygmy resonances in tin isotopes
}

\author{
E. G. Lanza, F. Catara, and D. Gambacurta \\ I.N.F.N.-Catania and Dipartimento di Fisica e Astronomia, Universitá di Catania, Via S. Sofia 64, I-95123 Catania, Italy \\ M. V. Andrés \\ Departamento de Física Atomica, Molecular y Nuclear, Universidad de Sevilla, Apdo 1065, E-41080 Sevilla, Spain \\ Ph. Chomaz \\ CEA, Irfu/Dir, Centre de Saclay, F-91191 Gif-sur-Yvette, France
}

(Received 19 January 2009; published 29 May 2009)

\begin{abstract}
We study, within a semiclassical coupled-channels approach, the possible effects of anharmonicities and non linearities on the excitation of the so-called pygmy resonances in several $\mathrm{Sn}$ isotopes and using two different Skyrme interactions. In the energy region of the pygmy resonances, there are a few low-lying multiphonon states. The question is whether they may contribute to the observed peak. Calculations show that the inelastic cross sections in the relevant energy region have an increase that varies from $3 \%$ to $21 \%$ depending on the isotope and on the kind of Skyrme force used. At the same time, we have studied the nature of the pygmy resonance state by means of a new criterion to establish whether this state can be considered as a collective one.
\end{abstract}

DOI: 10.1103/PhysRevC.79.054615

PACS number(s): 21.60.Ev, 21.60.Jz, 24.30.Gd, 25.60.-t

\section{INTRODUCTION}

In recent years, some evidence of new phenomena associated with increasing the neutron excess in nuclei has been accumulated [1]. In particular, approaching the neutron dripline, a neutron skin develops, i.e., a concentration of neutron density partly decoupled from the core nucleons. Oscillations of the skin neutrons against the core give rise to a low energy isovector mode: the pygmy dipole resonance (PDR). Dipole strength at low energies has been widely studied within several microscopic models, among which we quote the Hartree-Fock plus random-phase approximation (RPA) with Skyrme interactions, the relativistic RPA (RRPA) and the relativistic Hartree-Bogoliubov (RHB) plus the relativistic quasiparticle RPA (RQRPA). For a recent and up-to-date bibliography, see Ref. [1]. Whether or not such strength corresponds to a collective mode is still under discussion. In particular, when the spreading effects arising from the coupling of single nucleon states to the collective low-lying excitation are included within the QRPA plus phonon coupling model (QPM), the peaks associated with the PDR in several nuclei, both light [2] and heavy or medium-heavy [3,4], contain just a few neutron particle-hole configurations. These results are at variance with the findings of the RRPA plus phonon coupling model [5].

From the experimental side, evidence for Coulomb excitation of PDR in heavy ion collisions at high energies on ${ }^{132} \mathrm{Sn}$ has been reported [6,7] quite recently. In the same energy region, dipole states whose main components are two-phonon configurations are present [8] and effects coming from their possible mixing with the PDR can be envisaged. A similar question has been addressed in Refs. [4,9], in which the structure of the low-lying energy part of the dipole strength distribution was analyzed in these terms.

Thus it seems to us appropriate to study the excitation of the PDR in the framework of the semiclassical coupled-channel approach already used for the calculation of the multiple excitation of giant resonances in heavy ion collisions [10-12]. Within such an approach, anharmonicities and nonlinearities are treated in a completely microscopic description based on the HF plus RPA with Skyrme interaction, and they have been found to be responsible for an appreciable increase of the inelastic scattering cross sections. In particular, in Ref. [10] it was found that a kind of cooperative effect leads to a very strong enhancement of the Coulomb excitation cross section of a low-lying state in ${ }^{208} \mathrm{~Pb}$, whose main component is built with the low-lying $2^{+}$and $3^{-}$states coupled to a total $J^{\pi}=1^{-}$. For the tin isotopes that we are going to study, these states lie in the region of the pygmy resonances, and the question is whether they may contribute to the observed peak.

The paper in organized as follows. In Sec. II, we present the HF+RPA strength distributions for some closed shell Sn isotopes. In particular, an analysis about the properties of PDR is carried out by means of a novel criterion aimed at studying the features and the collectivity degree of the PDR. In Sec. III, the framework within which our calculations are done is briefly described (the details can be found in our previous work), while in Sec. IV, the results about inelastic cross section calculations are presented and discussed. Finally, in Sec. V, the main conclusions are drawn.

\section{RPA RESULTS}

In this section, we present the RPA strength distributions for the closed shell $\mathrm{Sn}$ isotopes ${ }^{100} \mathrm{Sn},{ }^{120} \mathrm{Sn}$, and ${ }^{132} \mathrm{Sn}$. We compare the results obtained with two Skyrme interactions, namely, the SGII [13] and the SLY4 [14]. We have chosen these particular Skyrme interactions, among the many known in the literature, in order to compare the results obtained with an interaction whose parameters have been fixed, paying a special attention to nuclei with a neutron excess (SLY4), and those 


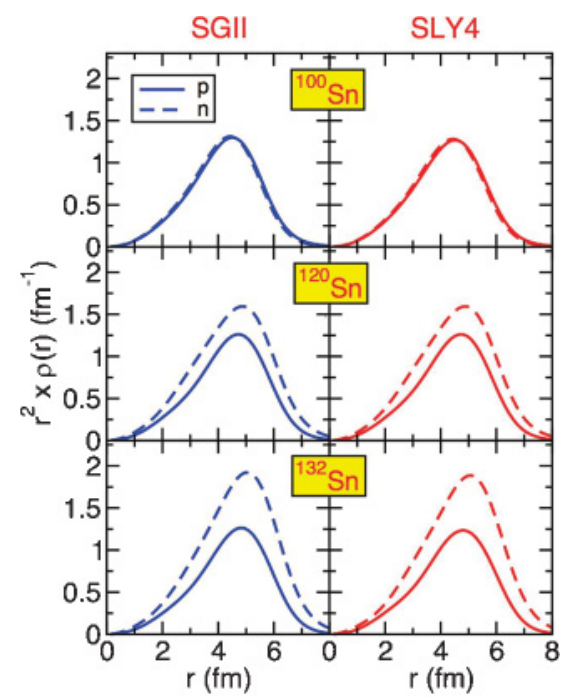

FIG. 1. (Color online) Proton (solid) and neutron (dashed) densities for three isotopes of Sn calculated with both SGII and SLY4 interactions.

coming out with an interaction widely used, also by us, and adjusted also on the properties of excited states (SGII). They are both representative of acceptable Skyrme interactions.

First, we solve the Hartree-Fock equations and express the single-particle wave functions as superpositions of 16 harmonic oscillator ones. In Fig. 1, we show the proton and neutron densities for the three isotopes ${ }^{100} \mathrm{Sn},{ }^{120} \mathrm{Sn}$, and ${ }^{132} \mathrm{Sn}$ and for the two considered interactions. They are multiplied by $r^{2}$ to make more evident the presence of the neutron skin. The results do not depend appreciably on the used interaction.

For the most neutron rich isotope, we see that the neutron density extends to larger radial values with respect to the proton density, while the opposite happens for ${ }^{100} \mathrm{Sn}$. This is quantified in the difference between the neutron and proton rms radii (see Table I). The values obtained with the SLY4, very similar to those calculated in Ref. [4], are slightly larger than the ones with the SGII. This is because the formation of the skin is related to the value of the nuclear symmetry energy [7,15,16], which is $32 \mathrm{MeV}$ [14] and $26.8 \mathrm{MeV}$ [13] for the SLY4 and SGII, respectively. A clear connection of the low energy $B(E 1)$ strength and the thickness of the neutron skin has been shown and its effect on the transition densities amply discussed in Refs. $[4,5,17,18]$. Below we will present, for ${ }^{132} \mathrm{Sn}$, a comparison between the transition density associated with the low-lying dipole state and that of the giant dipole resonance (GDR). As we will see, this comparison clearly indicates the different nature of the oscillations from which

TABLE I. Difference $\delta r$ (in fm) between the neutron and proton $\mathrm{rms}$ radii.

\begin{tabular}{lrr}
\hline \hline & \multicolumn{1}{c}{ SGII } & \multicolumn{1}{c}{ SLY4 } \\
\hline${ }^{100} \mathrm{Sn}$ & -0.086 & -0.100 \\
${ }^{120} \mathrm{Sn}$ & 0.119 & 0.142 \\
${ }^{132} \mathrm{Sn}$ & 0.198 & 0.221 \\
\hline \hline
\end{tabular}

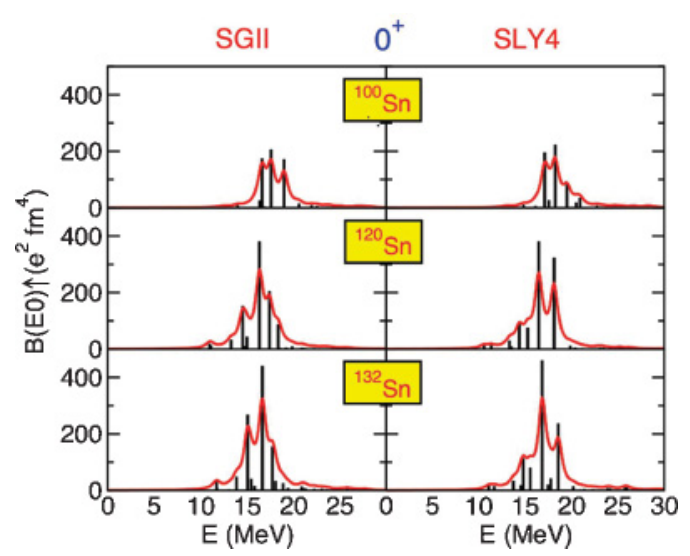

FIG. 2. (Color online) Isoscalar strength distributions for monopole states for tin isotopes calculated with the SGII and SLY4 interactions. The solid curves represent $d B(E 0) / d E$ in units of $\left(e^{2} \mathrm{fm}^{4} \mathrm{MeV}^{-1}\right)$ as obtained by adopting a smoothing procedure described in the text.

the two peaks originate and the fact that, indeed, the low-lying one can be identified with the pygmy dipole resonance (PDR). An exhaustive discussion of the evolution of the PDR and its identification can be found in Ref. [4], where the results for several $Z=50$ isotopes and $N=82$ isotones, within QRPA, are presented and analyzed. Other systematic studies on Sn isotopes have been reported also in Refs. [19,20].

In Figs. 2-5, we show a compendium of the RPA strength distributions for all the considered isotopes and the two Skyrme forces, for the natural parity multipolarities from $J^{\pi}=0^{+}$to $J^{\pi}=3^{-}$. The bars correspond to the RPA calculations, while the continuous lines are generated by a smoothing procedure using a Lorentzian with a $1 \mathrm{MeV}$ width. The continuous lines are drawn only to easily see where the major strength is located. The monopole states are not excited by the Coulomb interaction. As shown in Ref. [21], their presence is important in generating anharmonicities. Their strength distribution does not vary significantly going from one isotope to the other. In

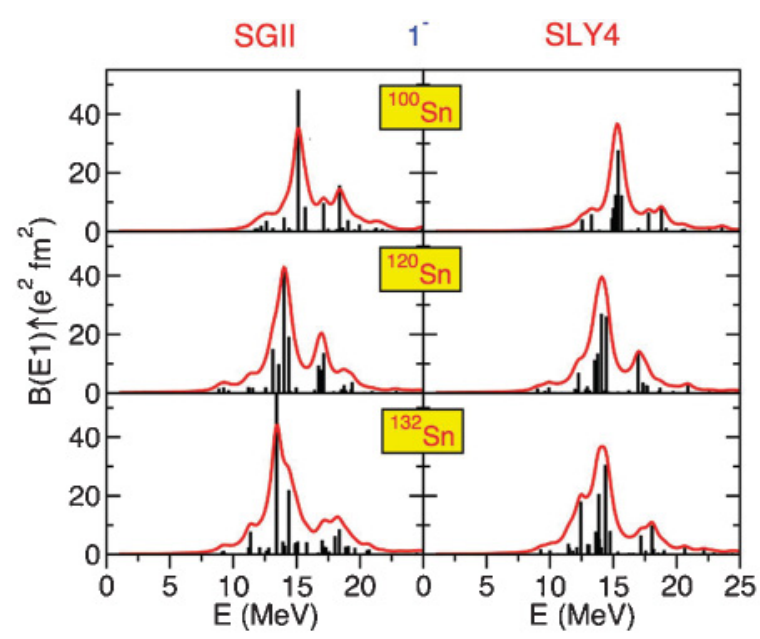

FIG. 3. (Color online) Same as Fig. 2, but for isovector strength distributions for dipole states. The solid curves are $d B(E 1) / d E$ in units of $\left(e^{2} \mathrm{fm}^{2} \mathrm{MeV}^{-1}\right)$. 


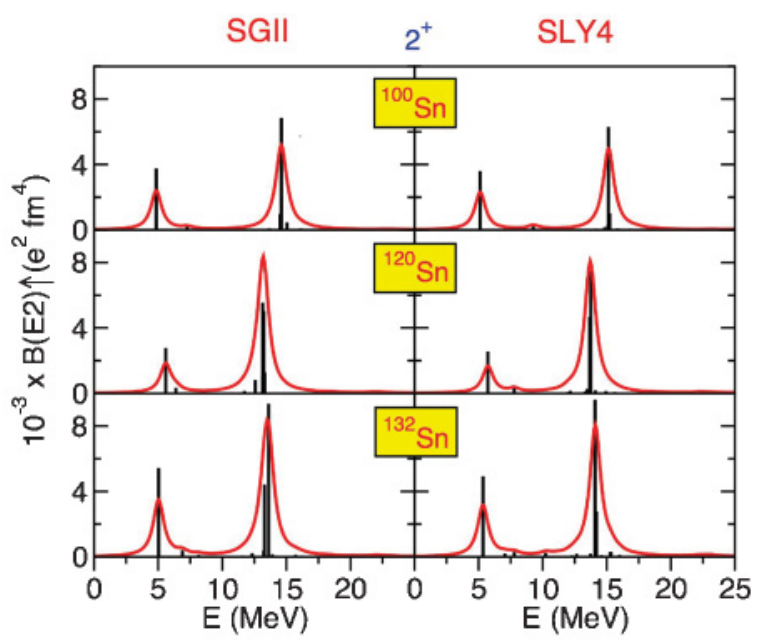

FIG. 4. (Color online) Same as Fig. 2, but for isoscalar strength distributions for quadrupole states. The solid curves are $d B(E 2) / d E$ in units of $\left(e^{2} \mathrm{fm}^{4} \mathrm{MeV}^{-1}\right)$.

Fig. 3, we see that, moving toward larger neutron excess, some dipole strength develops at low energies. This result is quite the same for the two interactions, the only difference being that the low-lying dipole strength is more fragmented with the SLY4 force. How much this strength can be interpreted as related to a pygmy resonance requires an analysis similar to that done in Refs. [4,5,17] and it will be presented below. A moderate increase of the low-lying quadrupole and octupole strengths with the neutron excess is also visible in Figs. 4 and 5. The very low-lying $3^{-}$state in ${ }^{120} \mathrm{Sn}$ is related to the fact that the $\mathrm{h}_{11 / 2}$ single-neutron state is very close to the last neutron-hole states.

We have analyzed the isospin character of the considered multipole states. While the dipole ones are almost purely isovector, states of higher multipolarities show a rather strong isospin mixing, especially for the isotopes with a thicker neutron skin. This effect is not new and has been extensively

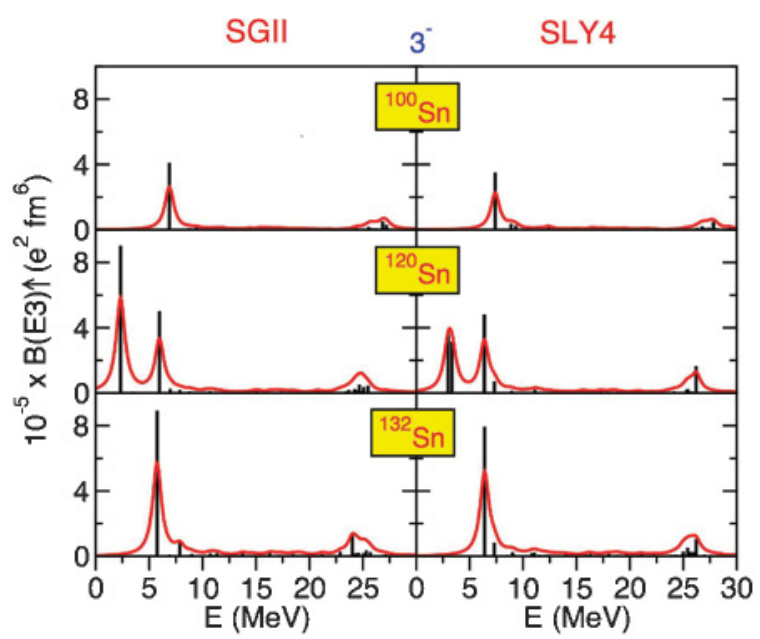

FIG. 5. (Color online) Same as Fig. 2, but for isoscalar strength distributions for octupole states. The solid curves are $d B(E 3) / d E$ in units of $\left(e^{2} \mathrm{fm}^{6} \mathrm{MeV}^{-1}\right)$.
TABLE II. Photoabsorption cross sections for the ${ }^{132} \mathrm{Sn}$ isotope calculated with the SGII and SLY4 interactions. In the third row we report the experimental values from Ref. [6]. The theoretical values of the cross section for the $1_{l l}^{-}$(GDR) were obtained by summing all the states below (above) $11 \mathrm{MeV}$.

\begin{tabular}{llcllc}
\hline \hline & \multicolumn{2}{c}{$1_{l l}^{-}$} & & \multicolumn{2}{c}{ GDR } \\
\cline { 2 - 3 } \cline { 5 - 6 } & $\begin{array}{c}E \\
(\mathrm{MeV})\end{array}$ & $\begin{array}{c}\int \sigma_{\gamma} \\
(\mathrm{mb} \mathrm{MeV})\end{array}$ & & $\begin{array}{c}E \\
(\mathrm{MeV})\end{array}$ & $\begin{array}{c}\int \sigma_{\gamma} \\
(\mathrm{mb} \mathrm{MeV})\end{array}$ \\
\hline SGII & 9.3 & 37 & & 13.92 & 2487 \\
SLY4 & 9.6 & 47 & & 13.81 & 2102 \\
Exp. & $9.8(7)$ & $75(57)$ & & $16.1(7)$ & $2330(590)$ \\
\hline \hline
\end{tabular}

discussed in the literature $[4,17]$. Such behavior is similar for the two used interactions.

The problem related to possible mixing of physical states with the spurious (isoscalar dipole) one associated with the center-of-mass motion deserves some discussion. It is very well known [22] that if one makes completely consistent $\mathrm{HF}+\mathrm{RPA}$ calculations, this state becomes a solution of RPA equations at zero energy, exhausting $100 \%$ of the energy weighted sum rule (EWSR). In our calculations, however, the spin-orbit term of the Skyrme interaction is neglected in RPA. This inconsistency is partially the origin of the fact that our lowest dipole state in ${ }^{132} \mathrm{Sn}$ with the SGII interaction, for example, is at $2.3 \mathrm{MeV}$. In the literature, a prescription suggested to cure this result is to multiply the $T=0$ component of the residual interaction in the $J^{\pi}=1^{-}$ channel by a factor such that the lowest dipole state is brought to zero energy [23]. We have done a few calculations by following such prescription and found that, with a factor of 1.1 , the energy goes down to $90 \mathrm{keV}$. On the contrary, the other dipole states, including the low-lying ones, are very marginally affected by this rescaling of the interaction. We can therefore conclude that the $T=0$ content in the latter states is small, and consequently, the mixing with the spurious state is small.

We have calculated the photoabsorption cross sections for the ${ }^{132} \mathrm{Sn}$ isotope in the two energy regions of interest: $E<$ $11 \mathrm{MeV}$ and $E>11 \mathrm{MeV}$. The results are shown in Table II together with the experimental values taken from Ref. [6]. The theoretical values have been obtained, for the two considered interactions, by using the expression [24]

$$
\int d E \sigma_{\gamma}=\frac{16 \pi^{3}}{9 \hbar c} \sum_{i} E_{i} B\left(E 1,0^{+} \rightarrow 1_{i}^{-}\right),
$$

where the sum is over the states belonging to the relevant intervals and the $B(E 1)$ 's include the effective charges $\frac{N}{A} e$ and $-\frac{Z}{A} e$ for protons and neutrons, respectively [25]. (Note that in Fig. 3 we plot the isovector reduced transition probabilities.) The agreement with the experimental data is good within the error bars, except for the position of the GDR peak, which is lower by $\sim 2 \mathrm{MeV}$.

\section{NATURE OF DIPOLE LOW-LYING STATES}

As already mentioned, one important question is how collective are the dipole states observed at low energies, which 
TABLE III. Particle-hole configurations that give the major contribution for two RPA dipole low-lying states for the nucleus ${ }^{132} \mathrm{Sn}$ and for the SGII and SLY4 interactions. For each p-h configuration, their energy, the contribution to the norm of the state $A_{\mathrm{ph}}$, and the partial contribution to the reduced transition amplitude $b_{\mathrm{ph}}$ (in $e$ fm units) are reported. The superscripts $\pi, v$ refer to the proton and neutron states, respectively.

\begin{tabular}{|c|c|c|c|c|c|c|c|}
\hline \multicolumn{4}{|c|}{ SGII $(E=9.34 \mathrm{MeV})$} & \multicolumn{4}{|c|}{$\operatorname{SLY} 4(E=9.27 \mathrm{MeV})$} \\
\hline p-h conf. & $E(\mathrm{MeV})$ & $A_{\mathrm{ph}}$ & $b_{\mathrm{ph}}(E 1)$ & p-h conf. & $E(\mathrm{MeV})$ & $A_{\mathrm{ph}}$ & $b_{\mathrm{ph}}(E 1)$ \\
\hline$\left(2 p_{3 / 2}, 2 d_{5 / 2}\right)^{\pi}$ & 9.62 & $1.8 \%$ & -0.42 & $\left(1 f_{5 / 2}, 1 g_{7 / 2}\right)^{\pi}$ & 11.47 & $1.0 \%$ & 0.42 \\
\hline$\left(1 g_{9 / 2}, 1 h_{11 / 2}\right)^{\pi}$ & 9.33 & $2.3 \%$ & 0.88 & $\left(2 p_{3 / 2}, 2 d_{5 / 2}\right)^{\pi}$ & 9.86 & $5.3 \%$ & -0.72 \\
\hline$\left(1 g_{7 / 2}, 2 f_{7 / 2}\right)^{v}$ & 9.12 & $4.5 \%$ & 0.06 & $\left(2 p_{1 / 2}, 3 s_{1 / 2}\right)^{\pi}$ & 10.50 & $1.9 \%$ & -0.16 \\
\hline$\left(2 d_{5 / 2}, 2 f_{7 / 2}\right)^{v}$ & 8.85 & $3.6 \%$ & -0.83 & $\left(1 g_{9 / 2}, 1 h_{11 / 2}\right)^{\pi}$ & 9.37 & $7.8 \%$ & 1.63 \\
\hline$\left(2 d_{3 / 2}, 3 p_{1 / 2}\right)^{v}$ & 9.04 & $14.9 \%$ & -0.65 & $\left(1 g_{7 / 2}, 2 f_{7 / 2}\right)^{v}$ & 8.88 & $2.2 \%$ & 0.05 \\
\hline$\left(2 d_{3 / 2}, 2 f_{5 / 2}\right)^{v}$ & 9.07 & $24.0 \%$ & 1.79 & $\left(2 d_{3 / 2}, 3 p_{1 / 2}\right)^{v}$ & 9.34 & $67.6 \%$ & 1.25 \\
\hline$\left(3 s_{1 / 2}, 3 p_{3 / 2}\right)^{v}$ & 9.09 & $43.9 \%$ & 1.47 & $\left(3 s_{1 / 2}, 3 p_{3 / 2}\right)^{v}$ & 10.07 & $4.1 \%$ & 0.41 \\
\hline$\left(3 s_{1 / 2}, 3 p_{1 / 2}\right)^{v}$ & 9.67 & $1.0 \%$ & -0.17 & $\left(3 s_{1 / 2}, 3 p_{1 / 2}\right)^{v}$ & 10.61 & $1.2 \%$ & 0.15 \\
\hline$\left(1 h_{11 / 2}, 1 i_{13 / 2}\right)^{v}$ & 9.21 & $1.7 \%$ & -0.88 & $\left(1 h_{11 / 2}, 1 i_{13 / 2}\right)^{v}$ & 10.01 & $4.9 \%$ & -1.47 \\
\hline
\end{tabular}

may be associated with the existence of a pygmy resonance. For very light nuclei (halo nuclei), the soft modes are not collective states, but rather they are generated by the radial extension of the very weakly bound single-particle neutron states [26]. One measure of the collectivity is the number of particle-hole components [2,5] entering in the RPA wave function with an appreciable weight. In our RPA calculations, we can analyze the nature of these low-lying states in more detail. As in Ref. [5], for a state $v$ the contribution of a particle-hole configuration can be calculated by defining the quantity

$$
A_{\mathrm{ph}}=\left|X_{\mathrm{ph}}^{v}\right|^{2}-\left|Y_{\mathrm{ph}}^{v}\right|^{2}
$$

in terms of the $X$ and $Y$ RPA amplitudes, with the normalization condition

$$
\sum_{\mathrm{ph}} A_{\mathrm{ph}}=1 .
$$

Then, one can see what the contribution is, in percentage, of a $\mathrm{p}$-h configuration to the formation of the state $v$. In Table III, we show such components for one low-lying dipole state (for the two considered interactions), together with their contribution to the norm of the states. Only the configurations contributing more than $1 \%$ are shown. Note that the major contribution comes from the neutron skin. Indeed, the neutron p-h configurations contribute $93 \%$ to the formation of the state in the SGII interaction, and $80 \%$ for the state obtained with the SLY4 interaction. For the SGII case, our result is very similar to the one obtained in Refs. [5,27], where a relativistic RPA was used for the study of the pygmy resonance. In fact, also in our case, several p-h configurations contribute with an appreciable weight. Conversely, in the SLY4 case, only one neutron component is dominating. This is similar to the results of Ref. [2].

The fundamental concept that underlies collectivity is coherence. The reduced transition probability from the ground state to the excited state $v$ can be written as

$$
B(E \lambda)=\left|\sum_{\mathrm{ph}} b_{\mathrm{ph}}(E \lambda)\right|^{2}=\left|\sum_{\mathrm{ph}}\left(X_{\mathrm{ph}}^{v}-Y_{\mathrm{ph}}^{v}\right) T_{\mathrm{ph}}^{\lambda}\right|^{2},
$$

where $T_{\mathrm{ph}}^{\lambda}$ are the $2^{\lambda}$ multipole transition amplitudes associated with the elementary p-h configurations. In Table III, we show the partial contributions $b_{\mathrm{ph}}$ for the considered states. The previous analysis, based only on the magnitude of $A_{\mathrm{ph}}$, can be misleading because it does not take into account the amplitude $T_{\mathrm{ph}}^{\lambda}$ nor the relative signs of the separate contributions. On the contrary, coherence plays a fundamental role in building a significant value for $B(E \lambda)$. Indeed, in Table III we see that by looking only at $A_{\mathrm{ph}}$, the SGII low-lying dipole state seems to satisfy the collective conditions. The values of $b_{\mathrm{ph}}$ seem to confirm this conclusion: the $\mathrm{p}-\mathrm{h}$ configurations with a higher percentage also have big $b_{\text {ph }}$ values. Conversely, $A_{\mathrm{ph}}$ values for the SLY4 state show a concentration of the strength essentially on one elementary configuration, washing out the hypothesis of collectivity. However, if we look also at the $b_{\text {ph }}$ values, we note that configurations with a small percentage may give big contributions to the reduced transition probability. With both interactions, one gets contributions from several configurations. It is to be noted, however, that quite strong cancellations lead finally to a not very large $B(E 1)$. This is clearly seen in Fig. 6, where the partial contributions $b_{\mathrm{ph}}$ vs the order number of the p-h configurations used in the RPA calculations for the two Skyrme interactions are plotted. The bars correspond to the individual $b_{\mathrm{ph}}$ values, while the continuous thin line is the cumulative sum of the contributions. The dotted lines divide the protons from the neutron configurations. The order goes from the most bound configurations to the higher ones. The figures on the left column are for the low-lying dipole states whose main characteristics are reported in Table III while the ones on the right column are the GDR states obtained with the two Skyrme interaction as indicated in the figure. In particular they correspond to the states at $E=13.4$ and $E=14.4 \mathrm{MeV}$. From the figure, we can clearly see how the $B(E 1)$ of the GDR states are built up by the small contributions of many $\mathrm{p}$-h configurations which add coherently. For the low-lying states, we have a different behavior: several p-h configurations participate in the formation of the $B(E 1)$, but some of them have opposite sign, giving rise to a final value that is small though, as we will see below, strong enough to get excited by 

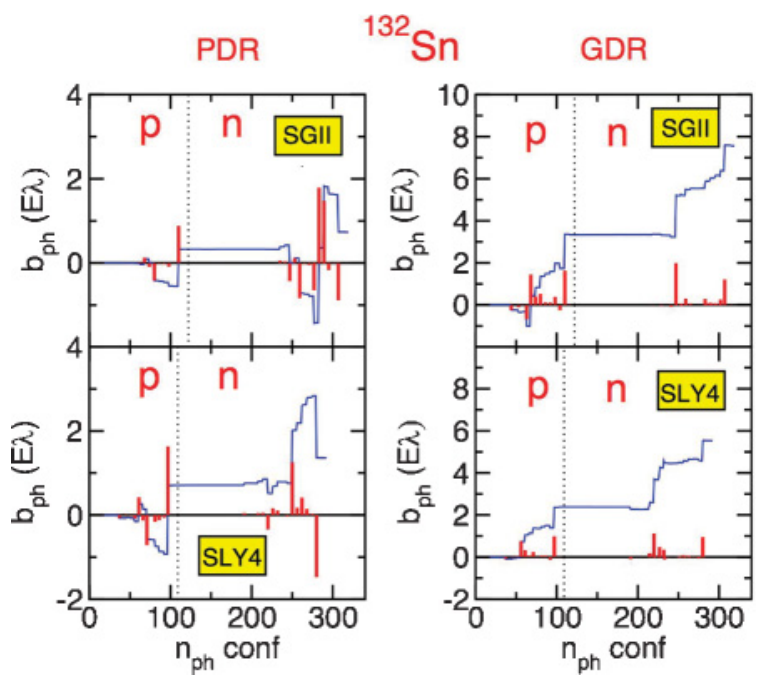

FIG. 6. (Color online) Partial contributions $b_{\text {ph }}$ of the reduced transition probability vs the order number of the $\mathrm{p}$-h configurations used in the RPA calculations for the two Skyrme interactions. The dotted lines divide the protons from the neutron configurations. The order goes from the most to the less bound ones. The bars corresponds to the individual $b_{\text {ph }}$ contributions; the continuous thin line is the cumulative sum of the contributions. The left column figures are for the two low-lying dipole states of Table III; the right column ones are for the GDR states.

the Coulomb interaction. From our novel analysis, it emerges that although the low-lying states cannot be considered as collective as the GDR states, they also cannot be described as a single $\mathrm{p}$-h configuration.

The same conclusion can be reached by looking at Fig. 7, where the RPA strength distributions as well as the unperturbed ones are reported for the ${ }^{132} \mathrm{Sn}$ isotope for the two interactions employed here. The bars are the RPA results, while the curves are obtained by the smoothing procedure described before. The dashed (black) lines represent the strength distributions for

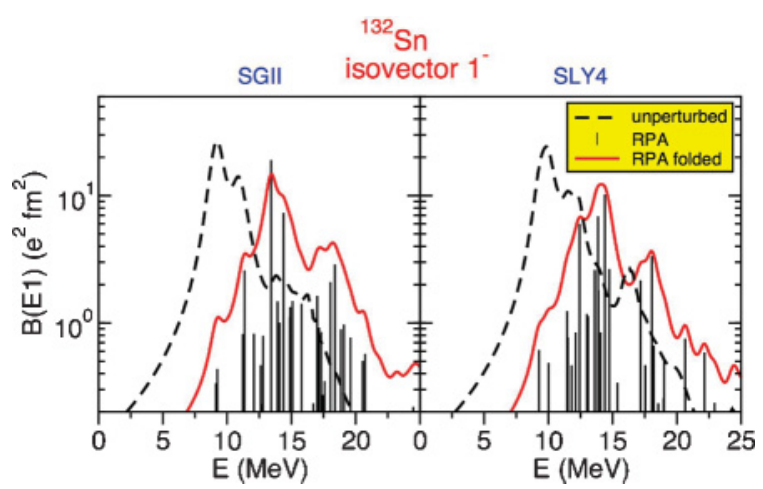

FIG. 7. (Color online) Strength distributions for dipole states for ${ }^{132} \mathrm{Sn}$ calculated with the SGII and SLY4 interactions. The bars show the RPA strength distributions. The curves represent $d B(E 1) / d E$ in units of $\left(e^{2} \mathrm{fm}^{2} \mathrm{MeV}^{-1}\right)$ as obtained by adopting a smoothing procedure (see text). The dashed (black) and solid (red) lines represent the unperturbed $\mathrm{p}-\mathrm{h}$ configurations and RPA smoothed strength distribution, respectively.

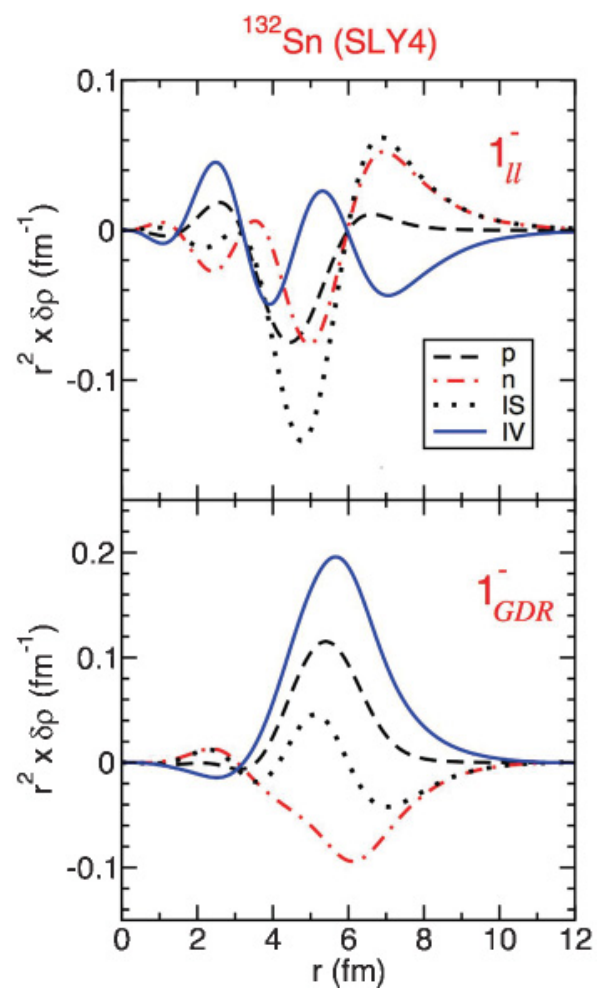

FIG. 8. (Color online) Transition densities for the low-lying dipole state (upper) and for the GDR (lower) for the ${ }^{132} \mathrm{Sn}$ isotope calculated with the SLY4 interaction. We show the proton, neutron, isoscalar, and isovector parts (as indicated in the legend).

the unperturbed $\mathrm{p}$-h configurations, while the solid (red) one corresponds to the RPA ones. When the residual interaction is switched on, the major part of the strength moves to higher energy, as it should be for an isovector state. The low-lying dipole states remain in their energy positions or move slightly to lower energy. The final RPA $B(E 1)$ values [Eq. (4)] are strongly reduced for the low-lying dipole states. Moreover, we remark that, as a consequence of the residual interaction, we observe in the low energy part of the strength distribution the appearance of some "structures" that are not present in the unperturbed case. They are related to the cooperative, although not coherent as discussed above, effect of several particle-hole excitations.

That the nature of the two dipole states discussed above is qualitatively different is illustrated in Fig. 8, where the proton, neutron, isoscalar, and isovector transition densities for the two states, calculated with the SLY4 interaction, are reported. The behavior for the low-lying state is similar to what has been found by several authors [3-5,17]. In the surface region, the proton and neutron densities are not out of phase, and the isoscalar transition density dominates over the isovector one; in the external region, only the neutrons give a contribution to both isoscalar and isovector transition densities which have the same magnitude. For the GDR case, we find the expected radial dependence for a collective isovector state: the proton and neutron transition densities oscillate out of phase; the isovector part is much larger than the isoscalar one for all distances beyond the radius of the nucleus. In addition, the 
isoscalar transition density shows some oscillations, which leads to a zero value for the corresponding $B(E 1)$.

In recent literature, there is a debate on the location of the PDR with respect to the neutron separation threshold. Many experiments have been done to measure the $E 1$ strength (i) below the threshold with $\left(\gamma, \gamma^{\prime}\right)$ studies $[8,18,28,29]$ for some $N=82$ isotones as well as for some $\mathrm{Sn}$ isotopes, (ii) just above the threshold with heavy ion collisions at the GSI $[6,7]$ for $\mathrm{Sn}$ isotopes, and (iii) in both regions with $(\gamma, x n)$ studies [30] for ${ }^{92,98,100}$ Mo isotopes. In both energy regions, there has been found clear evidence of $B(E 1)$ strength with a small percentage (about $1 \%$ ) of the $E 1$ isovector EWSR. From the theoretical point of view, we would like to mention selfconsistent RHB+RQRPA calculations of the peak excitation energy of the PDR as well as the one-neutron separation energy for a series of $\mathrm{Sn}$ isotopes [31]. The calculated one-neutron separation energy reproduce the experimental available data. The result show that for $A \geqslant 122$, the pygmy resonance is located above the neutron emission threshold; for smaller values of $A$, it is found below.

Nevertheless, whatever is the location of the low-lying dipole state, we want to stress once again that the only way to establish its nature, namely, that it is a different mode with respect to the GDR one, is to look at the transition densities. For example, in ${ }^{132} \mathrm{Sn}$ with the SGII interaction, the dipole strength distribution exhibits two small peaks below the GDR (see Fig. 3), at 9.34 and $11.36 \mathrm{MeV}$, both above the neutron threshold energy that we find equal to $8.56 \mathrm{MeV}$. However, their nature is completely different, as evidenced by looking at the transition densities in Fig. 9. In the lower part, we show the proton, neutron, isoscalar, and isovector transition densities for the dipole state at $E=11.36 \mathrm{MeV}$. Their pattern resembles very much the one of the GDR: protons and neutrons start to oscillate out of phase. Conversely, the behavior of the state at $E=9.34 \mathrm{MeV}$ is that of a "true" PDR state, i.e., similar to the one shown in the upper part of Fig. 8, although they have different strengths (note the different scale). So, although the strengths of the two states are very similar and their energies are close, they are two different nuclear modes as manifested in their different transition density behaviors.

\section{COULOMB EXCITATION CROSS SECTIONS}

Before starting the discussion about the calculations for the inelastic excitation cross section, we describe, very shortly, the framework within which these calculations have been done. The model has been developed for the study of multiple giant resonance excitations, and all the details can be found in Refs. [10-12].

The standard approach for studying multiple excitation of vibrational collective states implies the use of an internal harmonic Hamiltonian and an external excitation field, which is linear in the phonon creation and annihilation operators. Differences between theoretical standard calculations and experimental results have induced us to include corrections to the harmonic approximations, such as anharmonicities in the internal Hamiltonian and nonlinearities in the external field [10-12].

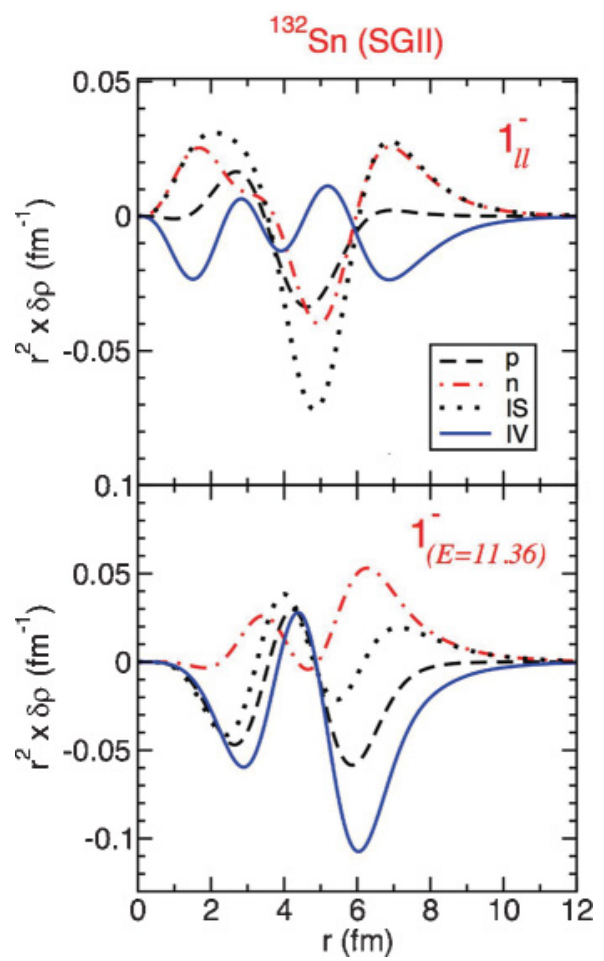

FIG. 9. (Color online) Transition densities for the low-lying dipole state (upper) and for the dipole state at $E=11.36 \mathrm{MeV}$ (lower) for the ${ }^{132} \mathrm{Sn}$ isotope calculated with the SGII interaction. We show the proton, neutron, isoscalar, and isovector parts (as indicated in the legend).

Studies of excitation processes of heavy nuclei have been achieved by using semiclassical methods techniques. These methods are based on the assumption that nuclei move on classical trajectories, while the internal degrees of freedom are treated quantum mechanically. These assumptions usually are well justified for grazing collisions (see Ref. [32]) and have also been applied to relativistic Coulomb excitations [33].

In these semiclassical models, the excitation of one of the partners of the collision is due to the mean field of the other. The excitation operator is of one-body type, and in standard models it is assumed to be linear in the phonon operator $Q$ as a consequence of the fact that only $\mathrm{p}$-h terms are taken into account. By using the boson mapping of Ref. [34] up to second order, and taking also the p-p and h-h contributions, we obtain a nonlinear excitation field

$$
\begin{aligned}
W= & W^{00}+\sum_{v} W_{v}^{10} Q_{v}^{\dagger}+\text { h.c. }+\sum_{\nu v^{\prime}} W_{v v^{\prime}}^{11} Q_{v}^{\dagger} Q_{v^{\prime}} \\
& +\sum_{v v^{\prime}} W_{v v^{\prime}}^{20} Q_{v}^{\dagger} Q_{v^{\prime}}^{\dagger}+\text { h.c. },
\end{aligned}
$$

which depends on time through the relative distance between the two nuclei. The first term in this equation represents the interaction of the two colliding nuclei in their ground state. The $W^{10}$ part connects states differing by one phonon, the $W^{11}$ term couples excited states with the same number of phonons, while $W^{20}$ allows transitions between states differing by two phonons. 
As stated above, the other missing ingredient is the anharmonicity in the internal Hamiltonian. Then, by adding to the RPA Hamiltonian the pppp, hhhh, ppph, and hhhp terms, which cannot be neglected in general, and by applying the boson mapping quoted above to the complete fermionic Hamiltonian, we construct a boson Hamiltonian

$$
H=H_{11}+H_{22}+H_{21}+\text { h.c., }
$$

where $H_{11}$ has a purely harmonic spectrum built with the RPA (multi)phonons, $H_{22}$ mixes states with the same number $(\geqslant 2)$ of phonons, and $H_{21}+$ h.c. mixes states whose phonon numbers differ by one.

From the results of the self-consistent $\mathrm{HF}+\mathrm{RPA}$ calculations, we select a reduced set of states that constitute our one-phonon basis by choosing states with angular momenta less or equal to 3 and with an appreciable percentage of EWSR. Then we construct all possible two- and three-phonon states out of them, and by diagonalizing the residual interaction in the space of one-, two-, and three-phonon states, we obtain the eigenstates of the Hamiltonian which are mixed states

$$
\left|\Phi_{\alpha}\right\rangle=\sum_{\nu} c_{v}^{\alpha}|\nu\rangle+\sum_{\nu_{1} v_{2}} d_{v_{1} \nu_{2}}^{\alpha}\left|v_{1} v_{2}\right\rangle+\sum_{\nu_{1} \nu_{2} v_{3}} e_{\nu_{1} \nu_{2} v_{3}}^{\alpha}\left|v_{1} v_{2} v_{3}\right\rangle
$$

whose corresponding eigenvalues are not harmonic. By expressing the state of the system in the presence of the excitation operator as a superposition of $\left|\Phi_{\alpha}\right\rangle$, the solution of the timedependent Schrödinger equation is cast into a set of coupled differential equations for the amplitudes which are integrated along the classical relative motion trajectories. For each $\left|\Phi_{\alpha}\right\rangle$, the associated cross section is obtained by integrating the excitation probability over the whole impact parameter range modulated by the transmission coefficient [11].

\section{RESULTS AND DISCUSSION}

To perform the calculations, we need to reduce the number of channels. Indeed, as can be seen from Figs. 2-5, several states for each multipolarity are present, some of them being much more collective than the others, which produces a spreading of the strength. Following the same prescription used previously [10], we put together the states in a given, small energy interval in such a way as to construct a single "doorway" state at an energy equal to the average value obtained by weighting the energy of the single states by their $B(E \lambda)$ and whose components are obtained with the constraint that it exhausts the fraction of EWSR given by the sum of the EWSR of the considered states. The so-obtained states are reported, for ${ }^{132} \mathrm{Sn}$, in Table IV for the two interactions. We construct all possible two- and three-phonon states out of these basis states, to build up the space where the bosonic Hamiltonian is diagonalized. If we consider that we have an equation for each angular momentum and its projection, we can easily realize that the number of time-dependent coupled equations to solve amounts to several thousands. To make feasible the cross section calculation, we took into account only the natural parity states with an excitation energy below $30 \mathrm{MeV}$. Furthermore, for each state, we considered in the
TABLE IV. RPA one-phonon basis for the nucleus ${ }^{132} \mathrm{Sn}$ and for both SGII and SLY4 interactions. For each state, the spin, parity, energy, and percentage of the EWSR (isovector for the GDR and the IVGQR, and isoscalar for all the other states) are reported.

\begin{tabular}{llrrrrr}
\hline \hline \multirow{2}{*}{ State } & \multirow{2}{*}{$J^{\pi}$} & \multicolumn{3}{c}{ SGII } & & \multicolumn{2}{c}{ SLY4 } \\
\cline { 3 - 4 } \cline { 6 - 7 } & & $\begin{array}{c}E_{\text {harm }} \\
(\mathrm{MeV})\end{array}$ & $\begin{array}{c}\text { EWSR } \\
(\%)\end{array}$ & & $\begin{array}{c}E_{\text {harm }} \\
(\mathrm{MeV})\end{array}$ & $\begin{array}{c}\text { EWSR } \\
(\%)\end{array}$ \\
\hline $0_{l l}^{+}$ & $0^{+}$ & 11.73 & 2 & & 11.36 & 2 \\
GMR & $0^{+}$ & 16.32 & 85 & & 16.87 & 86 \\
$1_{l l}^{-}$ & $1^{-}$ & 9.30 & 1.1 & & 9.60 & 1.4 \\
GDR & $1^{-}$ & 13.92 & 56 & & 13.81 & 55 \\
$1_{h l}^{-}$ & $1^{-}$ & 18.34 & 25 & & 17.75 & 15 \\
$2_{l l}^{+}$ & $2^{+}$ & 5.03 & 11 & & 5.35 & 11 \\
ISGQR & $2^{+}$ & 13.50 & 77 & & 14.11 & 70 \\
IVGQR & $2^{+}$ & 24.76 & 28 & & 24.03 & 36 \\
$3_{l l}^{-}$ & $3^{-}$ & 5.93 & 27 & 6.48 & 26 \\
HEOR & $3^{-}$ & 24.44 & 32 & 25.81 & 30 \\
\hline \hline
\end{tabular}

calculations only the components whose amplitude is larger than 0.03 . This still guarantees a very good normalization and reduces appreciably the computation time.

In Fig. 10, we present the cross section for the Coulomb excitation of ${ }^{132} \mathrm{Sn}$ in the collision with ${ }^{208} \mathrm{~Pb}$ at $500 \mathrm{MeV} / A$ incident energy. In the left part, we show the results for which the spectrum and multiphonon states (the channels) are those obtained with the SGII force. Of course, the cross section for exciting the GDR is dominating. Its intensity is a kind of gauge for the cross sections to the other states. The cross section to states up to $23 \mathrm{MeV}$ excitation energies are reported; however,

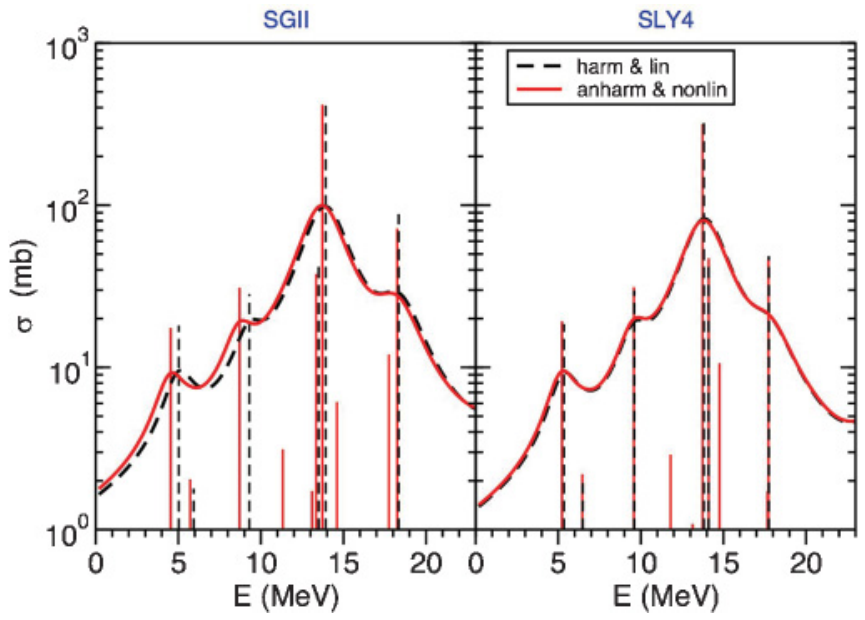

FIG. 10. (Color online) Relativistic Coulomb inelastic cross section for ${ }^{132} \mathrm{Sn}+{ }^{208} \mathrm{~Pb}$ at $500 \mathrm{MeV} / A$. The dashed black lines are the cross section obtained in the harmonic and linear approximation. The solid red lines correspond to the anharmonic and nonlinear case. The bars are the cross section for every single $\left|\Phi_{\alpha}\right\rangle$ state. The curves represent $d \sigma / d E$ in units of $\left(\mathrm{mb} \mathrm{MeV}^{-1}\right)$ and they are generated by a smoothing procedure using Lorentzian with a $2 \mathrm{MeV}$ width for the states whose energy is below $10 \mathrm{MeV}$ and with a $3 \mathrm{MeV}$ width for the ones with higher energy. These calculations were done with the SGII and SLY4 interactions. 
TABLE V. Same as Table IV, but for the nucleus ${ }^{120} \mathrm{Sn}$.

\begin{tabular}{lcccccc}
\hline \hline State & \multirow{2}{*}{$J^{\pi}$} & \multicolumn{3}{c}{ SGII } & & \multicolumn{2}{c}{ SLY4 } \\
\cline { 3 - 4 } \cline { 6 - 7 } & & $\begin{array}{c}E_{\text {harm }} \\
(\mathrm{MeV})\end{array}$ & $\begin{array}{c}\text { EWSR } \\
(\%)\end{array}$ & & $\begin{array}{c}E_{\text {harm }} \\
(\mathrm{MeV})\end{array}$ & $\begin{array}{c}\text { EWSR } \\
(\%)\end{array}$ \\
\hline GMR & $0^{+}$ & 16.43 & 85 & & 16.75 & 85 \\
$1_{l l}^{-}$ & $1^{-}$ & 9.21 & 1.8 & & 9.63 & 2.4 \\
GDR & $1^{-}$ & 13.90 & 50 & & 14.07 & 53 \\
$1_{h l}^{-}$ & $1^{-}$ & 16.97 & 22 & & 17.29 & 21 \\
$2_{l l}^{+}$ & $2^{+}$ & 5.68 & 8 & & 5.73 & 7 \\
ISGQR & $2^{+}$ & 13.20 & 80 & & 13.72 & 77 \\
IVGQR & $2^{+}$ & 24.57 & 37 & & 24.65 & 44 \\
$3_{1 l l}^{-}$ & $3^{-}$ & 2.33 & 12 & & 3.15 & 12 \\
$3_{2 l l}^{-}$ & $3^{-}$ & 5.96 & 17 & & 6.37 & 17 \\
HEOR & $3^{-}$ & 24.63 & 40 & & 25.94 & 36 \\
\hline \hline
\end{tabular}

all channels up to $30 \mathrm{MeV}$ were included in the calculations. Two series of results are shown, corresponding to two different levels of approximation. The dashed (black) lines represent the cross section obtained by considering the states in the harmonic limit and taking only the linear term of the external field. We see that, in this limit, only six states are excited when only the linear term of the field, $W_{10}$, is acting. They are pure one-phonon states. It is worth noting that the cross section associated with the pygmy resonance, at $9.30 \mathrm{MeV}$ excitation energy, is appreciable, being about $7 \%$ of the GDR. Let us now turn our attention to the continuous (red) lines corresponding to the case when anharmonicities and nonlinearities are not neglected. First, we notice that the GDR cross section is almost unaffected, passing from the $412 \mathrm{mb}$ value of the harmonic and linear limit to $416 \mathrm{mb}$ in the complete calculation, while for the PDR one gets 28 and $31 \mathrm{mb}$, respectively; and for the high-lying dipole state, the cross sections is reduced from 88 to $72 \mathrm{mb}$. We see also that the introduction of anharmonicity lowers the positions of the PDR and the low-lying $2^{+}$by $600 \mathrm{keV}$. Several more states are now excited, most of them are not visible in the figure because their individual cross section is below $1 \mathrm{mb}$. Among the visible ones, we mention in particular the state at $11.3 \mathrm{MeV}$, which in the harmonic limit is a pure $\left[3_{l l}^{-} \otimes 3_{l l}^{-}\right], J=2$ component, two-phonon configuration at $11.9 \mathrm{MeV}$. When the anharmonicities are switched on, it is pushed down and acquires a one-phonon GQR component, which is the second strongest one. Therefore the linear term of the field can excite it through such component. In addition, there is a contribution also from the other terms of the external field. In Fig. 11, a blow-up of the PDR region gives a better view of the effects we are talking about.

Similar analysis has been done for the same Sn isotope by using the SLY4 interaction, and the results are shown in the right side of Fig. 10. In the harmonic and linear limit, the main difference from the SGII results is that the value of the GDR cross section is quite smaller, $335 \mathrm{mb}$, while that of the PDR is almost unchanged, $30 \mathrm{mb}$, so that the ratio PDR/GDR is now $9 \%$. For the $1_{h l}^{-}$peak, we obtain a quite strong reduction to $49 \mathrm{mb}$. When anharmonicities and nonlinearities are included, several more peaks appear to be appreciably populated through the same mechanism discussed above. However, by inspection

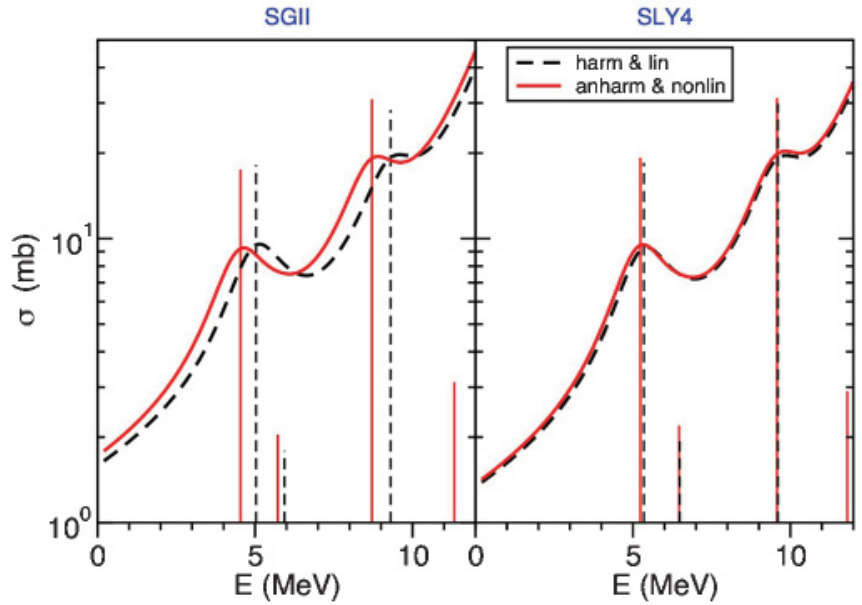

FIG. 11. (Color online) Blow-up of Fig. 10 in the PDR energy region.

of the figures, it is evident that the anharmonic effects are stronger in the SGII case. A more quantitative comparison can be done by looking at Table VI, which reports the cross sections summed over the energy intervals indicated in the first column. In particular, in the low energy interval (0-12 MeV), we have a $14 \%$ increase for the SGII case, while with the SLY4 force the increase amounts to $11 \%$. The peak at higher energy remains almost unchanged with a small reduction for the SLY4 calculations.

A similar analysis has been carried out for the system ${ }^{120} \mathrm{Sn}+{ }^{208} \mathrm{~Pb}$ at $500 \mathrm{MeV} / A$ (see Fig. 12). The overall behavior is very much the same as for ${ }^{132} \mathrm{Sn}$. Again, the dashed (black) lines refer to the harmonic and linear case and they correspond to the excitation of the one-phonon basis states, while the continuous lines are the results of the calculations when anharmonicity and nonlinearities are taken into account. As in the previous case, the calculations done with the SGII interaction show a stronger anharmonicity, as can be seen in Fig. 12. The summed inelastic cross sections are shown in Table VII. Apart from the difference in the absolute values,

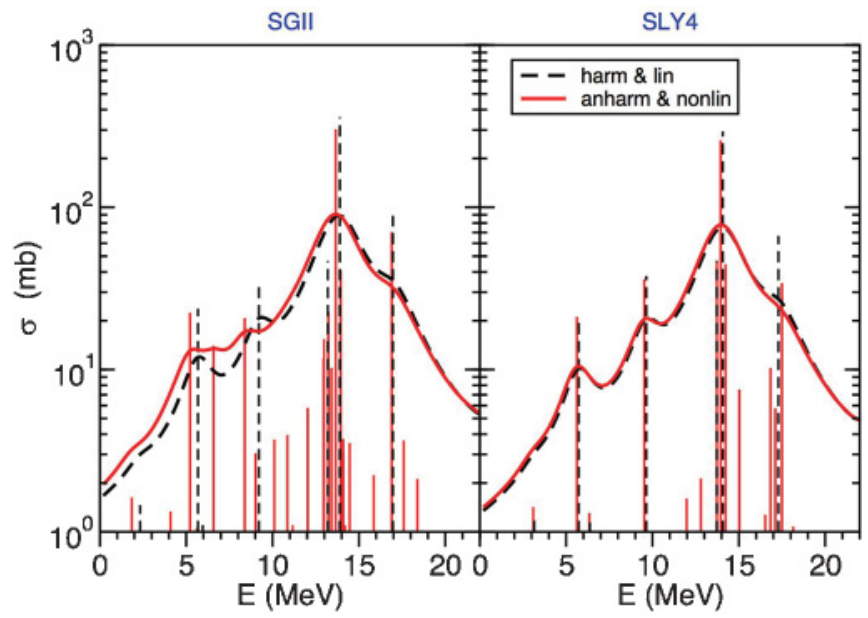

FIG. 12. (Color online) Same as Fig. 10, but for the system ${ }^{120} \mathrm{Sn}+{ }^{208} \mathrm{~Pb}$. 
TABLE VI. Summed inelastic cross sections for the system ${ }^{132} \mathrm{Sn}+{ }^{208} \mathrm{~Pb}$ at $500 \mathrm{MeV} / \mathrm{A}$ (for both SGII and SLY4 interactions) over the intervals shown in column one. Values for the harmonic and linear case (H-L) should be compared with those obtained in the anharmonic and nonlinear calculations (AH-NL).

\begin{tabular}{lccccr}
\hline \hline \multirow{2}{*}{$\begin{array}{l}c \\
\text { MeV }\end{array}$} & \multicolumn{2}{c}{ SGII } & & \multicolumn{2}{c}{ SLY4 } \\
\cline { 2 - 3 } \cline { 5 - 6 } & H-L $(\mathrm{mb})$ & AH-NL & & H-L (mb) & AH-NL \\
\hline $0-12$ & 48.3 & 55.0 & & 50.2 & 55.8 \\
$12-20$ & 543.9 & 547.0 & & 429.8 & 426.6 \\
\hline \hline
\end{tabular}

there is a stronger increase $(21 \%)$ for the SGII interaction than for the SLY4 case (only 3\%). This is essentially due to the strong excitation of the two-phonon state $\left[2_{l l}^{-} \otimes 3_{1 l l}^{-}\right], J=1$ component, which, in the SGII case, acquires by itself a cross section of $14 \mathrm{mb}$. This different behavior is associated with the fact that in the SGII case, when the anharmonicity is switched on, this state is shifted down in energy by $1.4 \mathrm{MeV}$ (going from 8 to $6.6 \mathrm{MeV}$ ), and it is strongly coupled with the $1_{l l}^{-}$state. Furthermore, in the ${ }^{132} \mathrm{Sn}$ case, there are a lot of multiphonon states, in the low-lying energy region, which obtain a considerable excitation when the anharmonicity is taken into account. Most of them are due to the presence of the $3_{1 l l}^{-}$which is strongly coupled to the other low-lying states. The use of the SLY4 interaction does not favor this coupling, so in the left part of Fig. 12 there is less contribution coming from the low-lying states.

As a conclusion of this work, we can certainly state that the inclusion of multiphonon states in the study of the PDR produces important effects, in particular when the SGII interaction is used. Indeed, we have found a considerable increase of the inelastic cross section for reactions with tin isotopes ${ }^{A} \mathrm{Sn}+{ }^{208} \mathrm{~Pb}$ at $500 \mathrm{MeV} / A$. The increase is relatively low with the SLY4 force with a remarkable difference as far as the anharmonicity behavior is concerned. Such a different response with respect to the anharmonicity is worth investigating, and it will be the argument of a future paper.

\section{SUMMARY}

In this work, we have extended the study of multiphonon excitations in heavy ion collisions to the case of the pygmy resonances. The motivation of this study relies on our findings on multiphonon investigation at low energy excitation. Indeed, we have found that a few low-lying multiphonon states are excited in the collision process with a quite strong probability. These states lay in the region of the pygmy resonances, and the

TABLE VII. Same as Table VI, but for the system ${ }^{120} \mathrm{Sn}+{ }^{208} \mathrm{~Pb}$.

\begin{tabular}{lrrrrr}
\hline \hline \multirow{2}{*}{$\begin{array}{l}c \\
\text { MeV })\end{array}$} & \multicolumn{2}{c}{ SGII } & & \multicolumn{2}{c}{ SLY4 } \\
\cline { 2 - 3 } \cline { 5 - 6 } & H-L (mb) & AH-NL & & H-L (mb) & AH-NL \\
\hline $0-12$ & 61.2 & 74.3 & 60.6 & 62.6 \\
$12-20$ & 496.0 & 502.9 & & 407.9 & 415.0 \\
\hline \hline
\end{tabular}

question is whether they may contribute to the observed peak. Starting from a microscopic approach based on RPA, mixing of two-phonon states among themselves and with one-phonon states is considered within a boson expansion approach with Pauli corrections. By diagonalizing a quartic microscopic Hamiltonian in the space of one-, two-, and three-phonon states, we generate mixed eigenstates with an anharmonic spectrum. Nonlinear terms are also taken into account in the external mean field of one of the nuclei which is responsible for the excitation of the other partner of the reaction.

The pygmy resonance is much more evident in nuclei with neutron skin, and it is thought to be generated by the oscillation of the neutrons in excess with respect to the core. To the discussion of whether this mode is a collective one, we have contributed with a novel analysis based on the investigation of the coherent construction of the reduced transition probability which should unambiguously give an answer to this problem. Our results show that although the degree of collectivity is not as high as the one of the GDR, they cannot be considered as due to one $\mathrm{p}$-h configuration. Indeed, while it is very well known that for the GDR many configurations contribute appreciably and add up coherently, in the PDR case there are several configurations contributing, but some of them cancel out so that, finally, the reduced transition probability $B(E 1)$ is quite smaller than what one should get without such cancellations and of the same order of a single p-h configuration.

The inelastic cross sections are calculated by solving semiclassical coupled-channel equations, the channels being superpositions of one-, two-, and three-phonon states. The calculations have been done for two different Skyrme interaction (SGII and SLY4) and for the reactions ${ }^{100,120,132} \mathrm{Sn}+{ }^{208} \mathrm{~Pb}$ at $500 \mathrm{MeV} / A$. We found an increase of the cross section in the low-lying PDR energy region, which is mainly due to the excitation of several states whose population is strongly suppressed by selection rules when anharmonicities and nonlinearities are neglected. The increase varies from $3 \%$ up to $21 \%$ depending on the isotope considered and the Skyrme force used. In general, the SLY4 interaction produces a smaller anharmonicity, which is then reflected in a lower increase of the cross section in the PDR region. In contrast, the results obtained with the SGII show the importance of anharmonicities and nonlinearities to the study of the PDR.

\section{ACKNOWLEDGMENTS}

We would like to thank the hospitality of the Departamento de Física Atómica, Molecular y Nuclear de la Universidad de Sevilla, where part of this work was done. This work makes use of results produced by the PI2S2 Project managed by the Consorzio COMETA, a project co-funded by the Italian Ministry of University and Research (MIUR) within the Piano Operativo Nazionale Ricerca Scientifica, Sviluppo Tecnologico, Alta Formazione (PON 2000-2006). More information is available at http://www.pi2s2.it and http://www.consorzio-cometa.it. This work has been partially supported by the Spanish Ministerio de Educación y Ciencia and the European regional development fund (FEDER) under 
project numbers FIS2008-04189 and FPA2006-13807-C0201; the Spanish Consolider-Ingenio 2010 Programme CPAN (CSD2007-00042) and Junta de Andalucía under project numbers P07-FQM-02894 and FQM160; and the bilateral agreement between the Spanish Ministerio de Educación y Ciencia and the Italian I.N.F.N., INFN08-31.
[1] N. Paar, D. Vretenar, E. Khan, and G. Colò, Rep. Prog. Phys. 70, 691 (2007) and references therein.

[2] G. Coló and P. F. Bortignon, Nucl. Phys. A696, 427 (2001).

[3] D. Sarchi, P. F. Bortignon, and G. Coló, Phys. Lett. B601, 27 (2007).

[4] N. Tsoneva and H. Lenske, Phys. Rev. C 77, 024321 (2008).

[5] D. Vretenar, N. Paar, P. Ring, and G. A. Lalazissis, Nucl. Phys. A692, 496 (2001).

[6] P. Adrich et al. (LAND-FRS Collaboration), Phys. Rev. Lett. 95, 132501 (2005); A. Klimkiewicz et al. (LAND-FRS Collaboration), Nucl. Phys. A788, 145 (2007).

[7] A. Klimkiewicz et al. (LAND Collaboration), Phys. Rev. C 76, 051603(R) (2007).

[8] U. Kneissl, N. Pietralla, and A. Zilges, J. Phys. G: Nucl. Part. Phys. 32, R217 (2006).

[9] N. Tsoneva, H. Lenske, and Ch. Stoyanov, Nucl. Phys. A731, 273 (2004)

[10] E. G. Lanza, M. V. Andrés, F. Catara, Ph. Chomaz, and C. Volpe, Nucl. Phys. A613, 445 (1997); A636, 452 (1998); A654, 792c (1999).

[11] M. V. Andrés, F. Catara, E. G. Lanza, Ph. Chomaz, M. Fallot, and J. A. Scarpaci, Phys. Rev. C 65, 014608 (2001).

[12] E. G. Lanza, F. Catara, M. V. Andrés, Ph. Chomaz, M. Fallot, and J. A. Scarpaci, Phys. Rev. C 74, 064614 (2006).

[13] N. Van Giai and H. Sagawa, Phys. Lett. B106, 379 (1981); N. Van Giai and N. Sagawa, Nucl. Phys. A371, 1 (1981).

[14] E. Chabanat, P. Bonche, P. Haensel, J. Meyer, and R. Schaeffer, Nucl. Phys. A635, 231 (1998).

[15] R. J. Furnstahl, Nucl. Phys. A706, 85 (2002).
[16] S. Yoshida and H. Sagawa, Phys. Rev. C 69, 024318 (2004).

[17] F. Catara, E. G. Lanza, M. A. Nagarajan, and A. Vitturi, Nucl. Phys. A614, 86 (1997); A624, 449 (1997).

[18] S. Volz et al., Nucl. Phys. A779, 1 (2006).

[19] J. Piekarewicz, Phys. Rev. C 73, 044325 (2006).

[20] J. Terasaki and J. Engel, Phys. Rev. C 74, 044301 (2006).

[21] M. Fallot, Ph. Chomaz, M. V. Andrés, F. Catara, E. G. Lanza, and J. A. Scarpaci, Nucl. Phys. A729, 699 (2003).

[22] P. Ring and P. Schuck, The Nuclear Many-Body Problem (Springer-Verlag, Berlin, 1980).

[23] M. Yamagami and Nguyen Van Giai, Phys. Rev. C 69, 034301 (2004).

[24] Aa. Bohr and B. R. Mottelson, Nuclear Structure, Vol. II (Benjamin, New York, 1975).

[25] M. N. Harakeh and A. van der Woude, Giant Resonances (Oxford University Press, USA, 2001).

[26] F. Catara, C. H. Dasso, and A. Vitturi, Nucl. Phys. A602, 181 (1996).

[27] E. Litvinova, P. Ring, and D. Vretenar, Phys. Lett. B647, 111 (2007)

[28] K. Govaert et al., Phys. Rev. C 57, 2229 (1998).

[29] A. Zilges, S. Volz, M. Babilon, T. Hartmann, P. Mohr, and K. Vogt, Phys. Lett. B542, 43 (2002).

[30] G. Rusev et al., Eur. Phys. J. A 27, 171 (2006).

[31] N. Paar, T. Nikšić, D. Vretenar, and P. Ring, Phys. Lett. B606, 288 (2005).

[32] R. A. Broglia and A. Winther, Heavy Ion Reactions (AddisonWesley, Reading, MA, 1991).

[33] A. Winther and K. Alder, Nucl. Phys. A319, 518 (1979).

[34] M. Hage-Hassan and M. Lambert, Nucl. Phys. A188, 545 (1972). 